recognised that many people are unsuitable for such treatment, particularly in the case of neurotic and highly reflex types;-so also individuals with blonde skins which do not easily pigment and easily freckle are not usually favourable subjects, and in these depression and not stimulation often follow the treatments. The same would also apply to all cases of hydroa æstivalis (recurrent summer eruption), in many of which there is an excess of hæmatoporphyrin in the urine. A condition of depression is also produced by an overdose in cases otherwise favourable.

It has been found that after exposure to light a rise in the hæmo-bactericidal power of the blood is demonstrable, which power persists for a few hours

\section{SOME NEUROLOGICAL CASE NOTES.}

\author{
BY
}

C. P. SYMONDS, M.D. OxF.,

ASSISTANT PHYSICIAN FOR NERVOUS DISEASES, GUY'S HOSPITAL ; ASSISTANT PHYSICIAN, NATIONAL HOSPITAL, QUEEN SQUARE.

\section{Tabes with Negative Wassermann Reaction in Blood and Spinal Fluid.}

A Man of 46 recently presented himself with the following story :

At the age of 21 he had a chancre. He was treated with mercurial pills for two years, and was then told by a high authority that he could regard himself as cured. A year later he married and subsequently had two healthy children. At the age of 36 he began to complain of a curious feeling of soreness in the skin of his chest and back, so that when bathing he had to go very gingerly in rubbing these parts with flannel or towel. At 39 he noticed that his lower limbs began to tire very easily and about the same time began to have attacks of shooting pains in the legs. These were of momentary duration, and at first trivial with long intervals of freedom. A year later he began to suffer from occasional attacks of epigastric pain and vomiting without relation to food, and from a gnawing pain in the rectum at times after passing a motion. He was then examined by a medical man, who, finding absent knee-jerks and pupils which reacted sluggishly to light, suggested the diagnosis of tabes. Further advice was sought and the Wassermann reaction done upon the blood and spinal fluid. In both it was negative. Various opinions were expressed, one being that the sluggish reaction of the pupils to light was a congenital abnormality and that the absence of knee-jerks was also congenital. He was given no treatment.

During the subsequent six years the symptoms continued and the pains were more severe. He lost his sexual powers and his sense of smell.

On examination the pupils were unequal and irregular, with hardly any reaction to light on the right side and very little on the left, the contraction in accommodation being very fair. The knee- and ankle-jerks were absent. There was extensive analgesia to pin-prick over the legs and trunk. Postural sensibility in the lower limbs was quite good and the gait normal.

When the Wassermann test was introduced it was at first supposed that a positive reaction was always obtained in tabes. For some years it has been recognised that this was a false opinion and and then returns to the normal. R. G. Bennerman oै has shown that an overdose of light, such as an initial exposure of half an hour to the mercury- 3 vapour lamp, produces a rise in the hæmo-bacteri- $\stackrel{\mathbb{Q}}{\stackrel{2}{2}}$ cidal power, which not only returns to normal, but $c$. which may fall below the original level and remain $\Rightarrow$ below it for some time before gradually rising to $\stackrel{0}{\rightarrow}$ the normal. This was found to be accompanied by certain other phenomena, notably an increase in $\frac{\bar{F}}{\bar{N}}$ the rate of sedimentation of blood corpuscles $\frac{\bar{\omega}}{\overrightarrow{0}}$

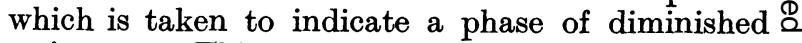
resistance. This apparent lowering of resistance $\%$ may account for the condition of depression which $\overrightarrow{0}$ is produced by the exposure to light of unsuitable cases.

that in some 20 per cent. of cases the test may be 3 . negative in blood and spinal fluid. This is more i likely to be so in cases in which the disease has $\vec{\omega}$ become spontaneously arrested or where the progress $\infty$ is slow. Negative reactions, therefore, should be $ᄋ$ discounted in the face of good clinical evidence. Tabes in its early stages is a disease of symptoms $O$ and the early symptoms are those of nerve-root $\frac{?}{0}$ irritation-pains and hyperæsthesiæ. The history given by this patient in the early stages of his illness is characteristic, and together with his story of a $\stackrel{\oplus}{N}$ syphilitic infection would have sufficed for the $\sigma$ diagnosis of tabes even in the absence of any physical signs.

The incidence of the disease upon the different groups of sensory fibres is variable. Quite commonly, as in the present instance, the pain $\stackrel{\mathbb{Q}}{\varnothing}$ fibres are affected without involvement of postural $\underset{\vec{A}}{\vec{B}}$ sensibility. There is, then, extensive cutaneous analgesia without ataxia.

The results of antisyphilitic treatment in tabes are difficult to assess owing to the variable course of the untreated disease. In the opinion of many observers a prolonged course of arsenical injections has a good effect: but the best that can be expected 3 is that the progress of the disease will be arrested. $\delta$ Hence the importance of early treatment. This should be given if symptoms are progressive $O$ whether the Wassermann reaction is positive or not. In stationary cases it is a wise plan to give a short annual course of four injections as a preventive measure.

Chronic Subdural Hamatoma.

A man of 19 was seen on June 18th with the following history :

Last February at a riding-school he had a fall from a horse, striking the back of his head. He suffered no $\Phi$ apparent injury and was able to catch his horse and $\stackrel{\mathscr{S}}{+}$ continue riding. He afterwards appeared dazed and had $T$ no clear memory of the events immediately following the $\frac{0}{\circ}$ fall. A few days later he began to have headaches. These $\underset{\mathbb{D}}{\mathbb{D}}$

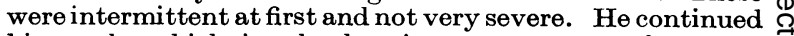
his work, which involved a journey to town from the $\mathbb{D}$ suburbs every day, and led an active life. During the general strike in May he rode his motor-cycle as a despatch rider.

On June 3rd he attended a dinner in town, at which he drank more than was good for him, and did not get to bed 
until the early hours of the morning. Next day he came to his doctor complaining of a splitting headache, which was attributed to the previous evening's festivities. In spite of the usual remedies, the headache was continuous with occasional severe paroxysms, some of them accompanied by vomiting. On June 16th it was noted that the optic discs were swollen, and his doctor first observed an extensor plantar response on the left.

When seen on June 18th he was lying in bed in severe pain, referred mainly to the right side of the head. The attempt to sit up induced giddiness and vomiting. Mentally he was clear. There was no aphasia. There was swelling of both optic discs, no defect of the visual fields. There was slight weakness of the lower part of the left face, and the palate moved to the right on phonation. There was no defect of sensation or of motor function. Reflexes were all normal, save for a definite extensor plantar response on the left. The pulse was 48. The head was normal on inspection and palpation, except for tenderness on percussion over the right frontal region.

A lumbar puncture was done immediately. The spinal fluid pressure was well above $300 \mathrm{~mm} . \mathrm{H}_{2} \mathrm{O}$ (the highest reading on the manometer). About $10 \mathrm{c.cm}$. of fluid was collected, but appeared to be blood-stained. A further quantity of $10 \mathrm{c.cm}$. was therefore received into a second tube. The result was a fall of pressure of $150 \mathrm{~mm} . \mathrm{H}_{2} \mathrm{O}$. Immediately afterwards the patient complained of a severe exacerbation of his headache.

On naked-eye inspection the fluid in the first tube was slightly hazy, due to the presence of a small quantity of "accidental" blood. That in the second tube was golden-yellow and slightly turbid from the presence of minute whitish flakes. The pathological report was that the first tube contained recent blood, probably due to accidental admixture at the time of puncture. The second tube showed no blood cells but 16 mononuclear cells per c.mm., 7 per cent. being large mononuclears. The flaky turbidity was due to the presence of fibrin. The protein was 0.04 per cent. The Wassermann reaction was negative.

At first sight one was inclined to discount the story of injury in this case and make the diagnosis of cerebral tumour. A point, however, which was unlike the story of a tumour, was the quite continuous nature of the severe headache from the day of its onset. This suggested the possibility of an acute syphilitic meningitis; and it was with the object of investigating this possibility further that lumbar puncture was performed.

Lumbar puncture in a case of increased intracranial pressure is never without risk; the cerebellum in such cases is forced down into the foramen magnum on top of the medulla, and removal of the supporting cerebro-spinal fluid from below may lead to a further increase of pressure upon the vital centres with fatal results. But here, balancing the value of the information to be gained by it against the risk, it appeared justifiable.

The intention was to remove not more than $10 \mathrm{c.cm}$. of fluid, but the presence of blood in the first tube made it necessary to collect another specimen. The fact that the second tube was clear proved that the blood in the first tube was due to accidental puncture of a vein. We were left, therefore, to explain the golden-yellow colour, the presence of fibrin in considerable quantity, and the slight increase in mononuclear cells. These abnormalities indicated old-standing hæmorrhage leaking into the subarachnoid space. The appearances suggested something of older standing than the headaches of a fortnight's duration, and one turned back again to the possibility of a chronic subdural hæmatoma dating from the injury.

It was decided that an operation should be performed. The left-sided extensor plantar response pointed to a lesion on the right side of the brain. When the bone was removed over the right temporal lobe the dura appeared normal. When it was opened the brain bulged out under considerable pressure. At the upper end of the opening, above the Sylvian fissure, the corner of a plum-coloured mass was evident. The bone being further removed in this direction, the dura mater in this region proved to be thickened and stained an ochreousyellow colour. Beneath it and quite separate from the dura lay a thin-walled cyst the size of a tangerine orange, which was full of bloody fluid darker than venous blood. The cavity was irrigated and drained and the prospect seemed good, but unfortunately the patient died of shock after the operation.

In these cases of chronic subdural hæmatoma the injury is often of a comparatively trivial nature, usually a blow or fall either upon the back or front of the head, the force of which is sufficient to rupture one of the small veins leading from the cortex into the superior longitudinal sinus. The effused blood, being contained within the two endothelial layers of the arachnoid membrane, does not clot, oozing continues slowly, and it may be some weeks before there are any serious signs of increased intracranial pressure. In this case the interval of four and a half months, during which the patient was leading an active life with only slight discomfort in the way of headaches, is remarkable.

\section{Disseminated Sclerosis beginning at the AGE of 50.}

The following case is that of a widow, aged 50 .

The patient complained to her doctor of a feeling of numbness and tingling in the right lower limb. It had first been noticed in the right thigh five weeks previously when it had only lasted a day or two. It then began again and spread this time downwards into the leg and upwards to the waist. With it was associated a feeling as if tight bands were wound about the limb above the ankle and knee. She was seen at this time and no physical signs were discovered. The diagnosis suggested was a neurosis.

The nature of the symptoms, however, was opposed to this. The hysterical patient seldom complains of numbness ; if she does, it will not be in these precise terms. The sensation of tight bands around the limb, in particular, is significant of spinal cord disease.

When she was examined two days later the numbness had spread to the left lower limb. There was diminished sensibility to cotton-wool and pin-prick over the lower limbs and trunk as far up as the axillæ, more marked on the right side than the left. The vibration sense was much diminished in the lower limbs. The right plantar response was extensor, the left absent. The knee- and ankle-jerks on both sides were brisk. The abdominal reflexes were lost.

So far the picture was one of a partial transverse lesion of the spinal cord at the level of the second dorsal segment. No other signs of nervous disease could be found except occasional nystagmoid jerking of the eyeballs on looking to the left. No history could be obtained of any nervous lesion in the past. 
The common causes of such a transverse lesion of the cord are compression from vertebral disease (growth or caries) or from a tumour of the meninges, syphilis, or a patch of disseminated sclerosis. In a young person the last named would have appeared at once the most likely cause. Nevertheless, in any case in which definite evidence of multiple lesions is lacking, the first two possibilities have to be excluded.

The spine showed no deformity, rigidity, or tenderness. An $\mathrm{X}$ ray plate of the cervical region revealed no signs of disease. The W.R. in blood and spinal fluid proved negative. The spinal fluid, however, in three respects was slightly abnormal. The cells numbered 6 per c.mm., a slight excess. The protein was 0.06 per cent., as compared with a normal figure of 0.02 per cent. The colloidal gold test gave a weak luetic curve. Although the fluid in a case of disseminated sclerosis is usually normal, this combination of slight abnormalities is not infrequently seen, especially if there has been a recent development of symptoms suggesting a fresh patch. A meningeal tumour sufficiently large to compress the spinal cord would probably occlude the subarachnoid space and so produce a considerably larger excess of protein.

Disseminated sclerosis is usually regarded as a disease of young people, and in the present instance the patient's age was a point against the diagnosis. But cases beginning in the fifth decade are by no means rare.

\section{Retro-Bulbar Neuritis due to Methylated SPIRITS PoIsoning.}

This case is a man of 40 , who was seen on account of recent loss of central vision.

Two months previously he had experienced sudden dimness of vision progressing in 24 hours to a bilateral central scotoma which cleared up completely in four days. After an interval of six weeks the trouble recurred and was associated with persistent vomiting which lasted several days. At the end of a fortnight the vision had not improved. On examination he had a large irregular central scotoma in each eye. Both optic dises were pale and the margins indistinct. There were no other signs of disease in the nervous system or elsewhere. . He denied the excessive use of alcohol or tobacco, his limits being two glasses of beer and six cigarettes a day.

In his past history there was a story of severe headaches five years previously. His blood at this time gave a positive Wassermann reaction, and the headaches were relieved after a course of potassium iodide. Nevertheless, he denied syphilitic infection.

The suggestion was that the recent visual trouble was a further expression of a latent syphilitic infection. On clinical grounds this appeared unlikely. Syphilis quite commonly causes optic atrophy and loss of vision, whether in association with disease of the brain or spinal cord, or as a single manifestation. But in these cases the onset is gradual and the defect of vision commences with a peripheral constriction of the visual fields. It is doubtful whether optic atrophy with central scotoma ever occurs as the result of syphilis. The Wassermann reaction proved negative in blood and spinal fluid; and the latter was normal in every respect. This did not in itself exclude syphilis as the cause, but supported the clinical argument against it. In the absence of any other cause, however, a course of antisyphilitic treatment was proposed. Before this was undertaken further search was made for a toxic origin and it. was eventually revealed that the patient had twice been treated for chronic alcoholism and had for the past year been secretly consuming an unknown quantity (at least $4 \mathrm{oz}$. daily) of methylated spirit. This substance contains 10 per cent. of methyl alcohol, which is known to be one of those poisons which have a selective action upon the papillo-macular bundle of fibres in the optic nerve causing optic atrophy with a central scotoma which may progress to complete blindness.

\section{THE THEORETICAL ASPECTS OF A C I D O I S.*}

BY

DUNCAN SCOTT, M.D.

(From the Physiological Laboratory, St. Bartholomew's Hospital.)

WE must begin with a consideration of the terms which it is necessary to understand before we can frame a definition of acidosis. Acids, alkalis, and salts are dissociable substances, the two companion ions of which they are formed parting company when they are in dilute solution. These companion ions are kations which carry a positive electric charge and anions carrying a negative charge. Acids owe their properties to the presence in their molecule of one or more hydrogen atoms, each of which can act as a kation when the acid is in watery solution, just as alkalis owe theirs to one or more hydroxyl ions $(\mathrm{OH})$, each with a negative charge. A "strong" acid is one in which dissociation occurs readily, so that its solutions possess a high proportion of free hydrogen ions, while "strong" alkalis similarly have many free hydroxyl ions. "Weak" acids and alkalis are much less readily dissociable. Pure distilled water contains both free hydrogen and free hydroxyl ions, but in it the numbers of both types of ions are the same and the fluid is consequently neutral in character.

\section{"Normat" Solutions.}

"Normal", solutions are solutions which contain the combining weight of the substance in 1 litre of solution. In quantitative chemistry we compare different strengths of the same acid and acids of different "strengths" in terms of normality; thus $10 \mathrm{c.cm}$. of normal acid, or $100 \mathrm{c.cm}$. of decinormal, or the arithmetical equivalent of this, whatever be the acid, will exactly neutralise $10 \mathrm{c.cm}$. of normal alkali. But in physical $\underbrace{\sigma}$ chemistry it is convenient to talk in terms of

* A Lecture given at the Children's Clinic, st. Bartholomew IIospital. 